\title{
Uso de Métodos Anticonceptivos y Embarazo en Adolescentes Atendidas en el Hospital de Concepción De La Sierra
}

\section{Contraceptive Methods and Pregnancy in Teenagers Who Received Services in Hospital of Concepcion De La Sierra}

\author{
Recibido 29 Jun. 2013/Enviado para Modificación 24 Sep. 2013/Aceptado 15 Oct. 2013
}

\author{
Laura Mónica Dubois 1
}

Hospital Concepción De La Sierra

\section{RESUMEN}

Introducción: El embarazo en la adolescencia responde a una causalidad muy variada y difícil de manejar y cambiar. En Argentina, la maternidad adolescente tiene una tasa alta en relación con la tasa general de fecundidad. Objetivo: Realizar un perfil de las pacientes menores de 24 años que se atienden en el Hospital de Concepción de la Sierra, además de recabar información acerca de su conocimiento de los métodos anticonceptivos. Materiales y métodos: Estudio prospectivo-descriptivo en 132 mujeres de 13 a 24 años. La técnica de recolección utilizada fue la encuesta, que permitió realizar un perfil de las mismas. Resultados: la edad promedio del grupo objeto de estudio fue de 18,4 años. El 29\% de las adolescentes culminó la educación primaria, y el $78 \%$ conocía la existencia de los métodos anticonceptivos, en tanto que el 75\% refirió usar o haber usado alguno. Entre los métodos más utilizados, se destacan las pastillas orales en un $71 \%$, el $80 \%$ de las jóvenes habían sido informadas acerca de su correcto uso. Al momento de la encuestas, $33 \%$ de las pacientes estaban embarazadas, pero el $41 \%$ de estas refirió no haber buscado el mismo. Por último, el $60 \%$ de las pacientes no embarazadas ya había tenido una gestación previa. Conclusiones: las encuestadas presentaron un bajo nivel educacional. Conocen los métodos anticonceptivos y la mayoría los usa o usó alguna vez. Al momento de aplicar la encuesta, más del $50 \%$ no estaba embarazada, aunque todas presentaban por lo menos una gestación previa, de las cuales la gran mayoría buscó el embarazo. Caso similar ocurrió con las pacientes embarazadas al momento de la encuesta.

Palabras Clave: Anticoncepción, adolescente, embarazo, métodos anticonceptivos. (Fuente: DeCS).

\section{ABSTRACT}

Introduction: The pregnancies correspond to a causality difficult to be managed and changed. In our country, teenage pregnancy is at a very high rate in relation to the rate of fertility. Objectives: To get a profile of patients under age 24 who are receiving services in Concepcion de la Sierra Hospital and to research information about their knowledge about contraception methods. Materials and Methods: Descriptive and prospective study taking a sample of 132 women from 13 to 24 years old. The tool used was a survey, which gave us a profile of those women. Results: The average age was 18.4 years old. 29\% finished primary school studies, $78 \%$ knew contraceptive methods, $75 \%$ informed to have used or used some contraceptive method. The most used method are oral pills at $71 \%$ and $80 \%$ of women were informed about correct use of that method. At the moment of the surveys, $33 \%$ of patients were pregnant. $41 \%$ did not inform to find the same method. $60 \%$ of the patients had had a previous pregnancy. Conclusions: The surveys show a low educational level. They know the contraception methods and most of them use or used those methods at the time which the survey was done. More than 50\% were not pregnant, although all of them presented at least a previous pregnancy and most of them wanted the pregnancy. A similar situation happened with pregnant patients at the time of the survey.

Keywords: contraception, adolescent, pregnancy, contraceptives methods (Source: MeSH, NLM).

1 Farmacéutica. Servicio de Farmacia del Hospital Nivel I de Concepción de la Sierra, Bartolomé Mitre y Sáenz Peña, Concepción de la Sierra, Misiones. e-mail: laur1801@gmail.com 


\section{Introducción}

Las adolescentes (10-19 años, según la OMS) y adolescentes tardías (20-24 años) (1) constituyen un campo de estudio de gran importancia, porque se hallan en la edad en que se inicia la vida sexual activa. Además, debido al cambio radical y definitivo en los conceptos de vida, familia y sociedad, la adolescencia es una época de transición en todos los aspectos: social, psicológico, físico y sexual. Por otra parte, aunque esta se considera una etapa de la vida de plena salud, durante este periodo puede existir una morbilidad excesiva (2).

El embarazo adolescente es un gran problema sanitario y social (3), no solo por el mayor riesgo que presentan las madres adolescentes de tener resultados perinatales y maternos desfavorables, sino también por toda la problemática familiar y de pareja que rodea a la adolescente (4).

En esta etapa de la vida, el embarazo responde a una causalidad muy variada y compleja, difícil de manejar y cambiar. Entre muchos factores que actúan, el inicio precoz de las relaciones sexuales, la información insuficiente sobre sexualidad y prevención de embarazo, el uso inadecuado o el no uso de métodos anticonceptivos, se encuentran entre los más importantes (5).

En Argentina, la maternidad adolescente presenta una tasa alta en relación con la tasa general de fecundidad, al punto de que el $14 \%$ de los niños que nacen en este país tiene una madre menor de 19 años, mientras que la población adolescente constituye el $30,6 \%$ de la población total. De esta última, el 49,9\% son mujeres (6). De manera más concreta, en Concepción de la Sierra, el 30,7\% de la población está constituida por adolescentes, cuya edad comprende entre 10 y 24 años, siendo el 51,1\% mujeres, y la tasa de fecundidad se corresponde con la nacional.

En este marco, mediante el presente trabajo, se pretende realizar un perfil de las pacientes menores de 24 años que se atienden en el Hospital de Concepción de la Sierra; recabar información acerca del conocimiento que tienen las adolescentes de los distintos métodos anticonceptivos; identificar el medio por el cual las adolescentes son informadas acerca de los métodos anticonceptivos y su correcto uso; y proponer sugerencias, de acuerdo con los resultados, que sirvan para mejorar el conocimiento y el uso correcto de los métodos anticonceptivos por parte de las adolescentes y sus parejas.

\section{Materiales y Métodos}

Se realizó una investigación observacional descriptiva, interrogando, para esto, a mujeres de entre 13 y 24 años, que asistieron a consulta durante los meses de Diciembre de 2011 y Enero y Febrero de 2012, en el Hospital de Concepción de la Sierra, Misiones, independientemente de si se encontraban embarazadas o no, pero excluyéndose a las que no habían tenido gestas previas.

La técnica de recolección utilizada fue la encuesta, y de acuerdo con los objetivos propuestos, se elaboró un cuestionario, mediante el cual se realizó la entrevista de manera anónima. Todas las encuestas fueron realizadas en el Servicio de Farmacia, al cual las pacientes acudían en busca de la medicación solicitada luego de la consulta. La técnica de procesamiento de la información fue computacional y se realizó la revisión y revalidación de la misma. Como medida de resumen de la información, se utilizó el porcentaje.

Los resultados se presentaron en tablas, que se analizaron para emitir las conclusiones, en correspondencia con los objetivos propuestos en la investigación.

\section{Resultados}

Fueron encuestadas un total de 132 mujeres de entre 13 y 24 años, que corresponden a cerca de $2,5 \%$ de la población atendida trimestralmente en dicho Hospital, ya que habitualmente se atienden alrededor de 5200 consultas trimestrales.

Respecto a la edad de las pacientes entrevistadas, el promedio fue de 18,4 años. La Tabla 1 muestra los distintos porcentajes, de acuerdo con las edades de las pacientes.

Tabla 1. Edades y porcentajes

\begin{tabular}{cc}
\hline EDADES & \% \\
\hline 13 & $2 \%$ \\
14 & $4 \%$ \\
15 & $9 \%$ \\
16 & $10 \%$ \\
17 & $10 \%$ \\
18 & $13 \%$ \\
\hline
\end{tabular}




\begin{tabular}{cc}
\hline EDADES & $\%$ \\
\hline 19 & $8 \%$ \\
20 & $12 \%$ \\
21 & $11 \%$ \\
22 & $10 \%$ \\
23 & $7 \%$ \\
24 & $4 \%$ \\
\hline
\end{tabular}

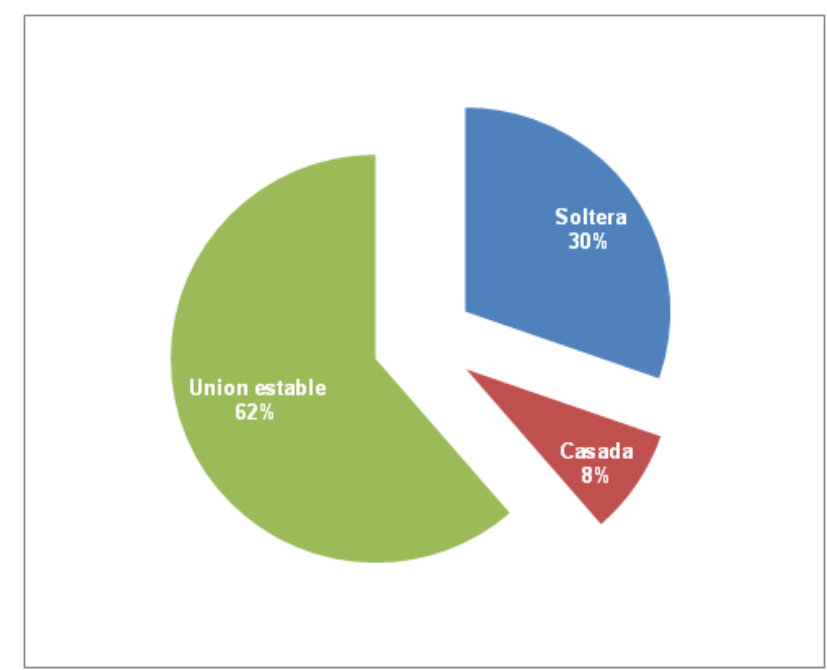

Figura 1. Estado civil de las pacientes encuestadas

La educación constituía un tema importante de evaluar en este trabajo, ya que se deben considerar distintas estrategias de educación a implementar en el futuro. La Figura 2 muestra los distintos niveles educacionales del grupo estudiado. En ella se percibe que el $29 \%$ terminó la escuela primaria, y que un idéntico porcentaje no completó ese nivel de estudio.

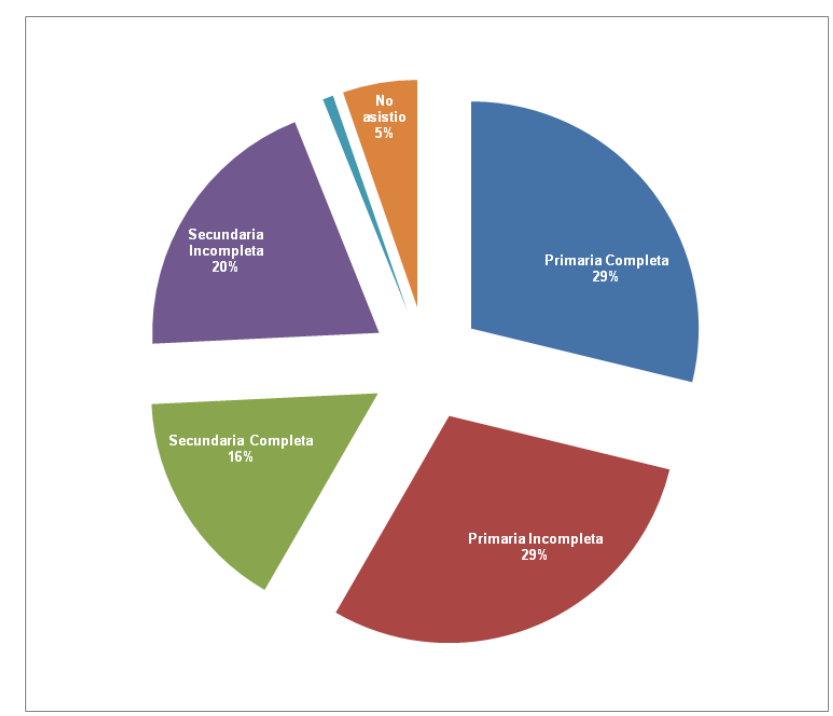

En cuanto a la cobertura social, solamente el $11 \%$ de las jóvenes refirió poseer un tipo de cobertura.

La Tabla 2 muestra la condición de vivienda que habitan, sea esta propia, prestada o alquilada.

Tabla 2. Condición de vivienda

\begin{tabular}{lc}
\hline \multicolumn{1}{c}{ VIVIENDA } & \% \\
\hline Propia & $62 \%$ \\
Propia (padres) & $3 \%$ \\
Prestada & $21 \%$ \\
Alquilada & $14 \%$ \\
\hline
\end{tabular}

También se consideró si tenían acceso a agua potable y a luz eléctrica, y la cantidad de personas que vivían en la misma vivienda. Al respecto, se encontró que el $81 \%$ tenía luz eléctrica en su domicilio y que el $77 \%$ poseía acceso al agua potable. Mientras que para los menores de 21 años que habitaban la vivienda, el promedio fue de 2,7 personas y mayores de 21 años, 1,9 .

En cuanto al conocimiento de los distintos métodos anticonceptivos, el $78 \%$ de las pacientes encuestadas conocía la existencia de los mismos; el $75 \%$ refirió usar o haber usado alguno. Entre los métodos anticonceptivos más utilizados, se destacan las pastillas orales con un $71 \%$, seguido de los preservativos con un $13 \%$ (Figura 3). El $78 \%$ de las pacientes que usaron o usan algún método anticonceptivo lo obtienen del Hospital en forma gratuita.

De las pacientes, el $80 \%$ había sido informada acerca del correcto uso, mientras que el $20 \%$ restante, no. En cuanto a las adolescentes que habían recibido educación acerca del uso de los métodos anticonceptivos, el $72 \%$ la recibió del médico prescriptor; el $8 \%$, del personal de enfermería; y $5 \%$, de sus familiares.

\section{Figura 2. Nivel Educativo}

Cienc. innov. salud. Diciembre 2013; 1 (2):114 - 119. Universidad Simón Bolívar (Col). ISSN: 2344-8636 http://portal.unisimonbolivar.edu.co:82/rdigital/innovacionsalud 


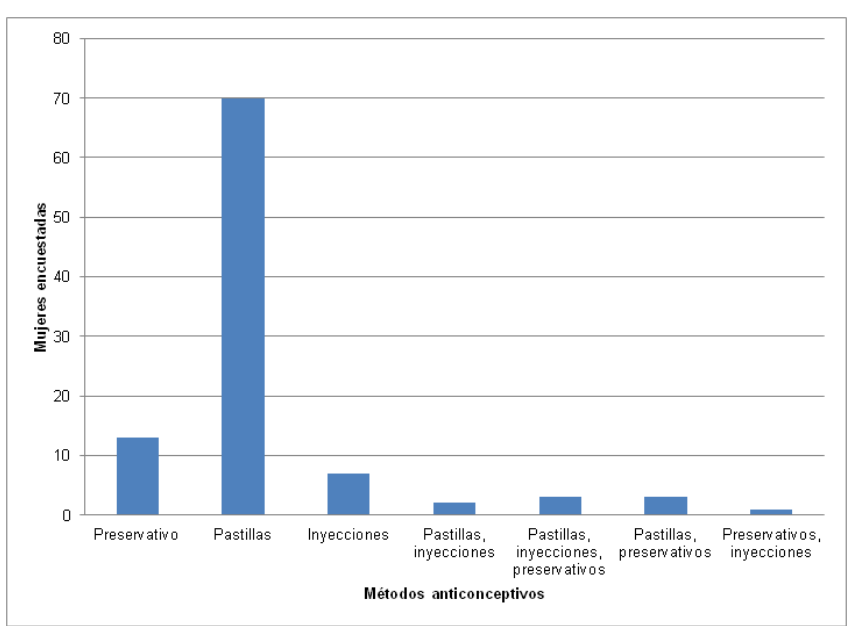

Figura 3. Métodos anticonceptivos empleados por mujeres encuestadas

Tabla 3. Embarazadas encuestadas

\begin{tabular}{ll}
\hline \% Embarazadas & Paridad \\
\hline & $43 \%$ primigestas \\
$33 \%$ embarazadas & $30 \%$ secundigestas \\
& $18 \%$ tercera gesta \\
& $9 \%$ cuarta gesta \\
\hline & $60 \%$ una gesta previa \\
& $26 \%$ dos gestas previas \\
& $11 \%$ tres gestas previas \\
& $3 \%$ cuatro gestas previas \\
\hline
\end{tabular}

Al momento de aplicar las encuestas, $33 \%$ de las pacientes estaban embarazadas y las restantes, no (67\%), observándose además la paridad de las mismas (Tabla 3).

De las pacientes no embarazadas, el $60 \%$ refirió haber buscado el embarazo; el $40 \%$ restante, no. De estas, el $76 \%$ dijo no haber usado método anticonceptivo alguno, y el $24 \%$ afirmó que había fallado el método.

En forma similar, el $59 \%$ de las pacientes embarazadas al momento de la encuesta dijo haber buscado el embarazo, mientras que el $41 \%$ no lo buscó. De estas últimas, el 33\% refirió alguna falla en el método anticonceptivo y el $67 \%$ no usó ninguno. Analizando los ingresos de las pacientes encuestadas, se puede observar que, en la mayoría de los casos, provienen de la asignación universal por hijo (42\%), seguido del trabajo rural (14\%), empleada doméstica y albañilería (11\%), changas (8\%).

\section{Discusión}

Las adolescentes que acuden habitualmente la consulta tienen en promedio 18 años, lo que no se relaciona con lo expuesto por De Dios en estudios realizados en países vecinos $(5,7)$, donde el promedio de edad es inferior. Del $100 \%$ de las pacientes encuestadas, el $30 \%$ son solteras, y el $62 \%$ tiene una unión estable, lo que también contradice a De Dios en Uruguay y a Pomata en Argentina, donde más del $40 \%$ de las adolescentes son solteras y deben afrontar el embarazo sin pareja $(2,5,6)$. El hecho de que se tenga una pareja estable es un dato para tener en cuenta, pues sugiere la existencia de un vínculo familiar, que indica la presencia de apoyo psicológico y económico.

En cuanto a la educación, el 29\% completó la enseñanza primaria y el $16 \%$, la secundaria; sin embargo, otro $29 \%$ no completó el nivel primario y tampoco un $20 \%$, el nivel secundario. El tema educativo es de suma importancia, ya que, para implementar cualquier medida de salud, se debe conocer el nivel educativo de los destinatarios. En específico, se estaría ante una población encuestada con un bajo nivel educativo, debido a que, en su gran mayoría, no alcanzó un nivel de estudio superior al primario. Esta variable coincide con estudios realizados por De Dios y Molina $(5,7)$ en diferentes trabajos nacionales e internacionales, en los cuales se observa que el embarazo en la adolescencia implica una mayor deserción escolar $(5,7,8)$, como ocurrió en el presente estudio, en el que muchas adolescentes afirmaron que buscan el embarazo para abandonar el colegio y la casa paterna.

En consideración a si la vivienda en la que habitan es propia, se encontró que así ocurre en el $62 \%$ de los casos, viviendo en ellas un promedio de 2,7 menores de 21 años y 1,9 mayores de 21 años. Del mismo modo, se observó que el $81 \%$ tiene acceso a luz eléctrica y el $77 \%$ a agua potable, lo cual no se correlaciona con otros estudios en los que se encontró que los niveles de pobreza son extremos. En este caso, necesidades básicas de subsistencia están mayormente cubiertas las (2). La gran mayoría de las adolescentes encuestadas $(78 \%)$ conocía la existencia de los diferentes métodos anticonceptivos, tal como lo expone González en su estudio (9), por lo que llama la

Cienc. innov. salud. Diciembre 2013; 1 (2):114 - 119. Universidad Simón Bolívar (Col). ISSN: 2344-8636 
atención la alta tasa de embarazo. Si se conocen los métodos anticonceptivos, cabe preguntarse: ¿no son usados? ¿Se usan de manera incorrecta?

Principalmente, las adolescentes usan o usaron alguna vez pastillas anticonceptivas orales, las cuales son suministradas en forma gratuita por Salud Pública; siendo también este el método más utilizado en el estudio realizado por Cruz (10).

El 80\% de las pacientes recibió información sobre el correcto uso de los métodos anticonceptivos. De estas, el $72 \%$ la recibió del médico prescriptor y solo el $5 \%$ de sus familiares, lo que difiere de la bibliografía revisada, pues, según los resultados obtenidos por Pérez (11), la información se provee mayormente en la escuela.

Del total de pacientes entrevistadas, $33 \%$ estaban embarazadas al momento de responder la encuesta. En cuanto a estas, $43 \%$ eran primigestas y $30 \%$ presentaba su segunda gestación.

El restante $67 \%$ del total de pacientes entrevistadas no estaban embarazadas, pero se destaca que el $60 \%$ de las mismas ya había tenido una gestación previa; el $26 \%$, dos; y solo un $3 \%$, tres gestas previas (5).

De las pacientes embarazadas, la mayoría refirió haber buscado el embarazo, no haber usado un método anticonceptivo, pero en algunos casos también había ocurrido una falla en el mismo, lo que coincide con resultados reportados por otros autores $(12,13)$. Como en el caso anterior, el $60 \%$ de las pacientes no embarazadas al momento de la encuesta afirmó que había buscado el embarazo; y el $40 \%$ restante, no. De estas, el $76 \%$ dijo no haber usado método anticonceptivo alguno y el $24 \%$ aseguró que el método había fallado, como en lo expuesto por Díaz (1).

Llama la atención el alto porcentaje de embarazos planeados, considerando la corta edad de las pacientes, por lo que reconocemos que es necesario profundizar en el ámbito familiar de las mismas, ámbito poco explorado, para así conocer en detalle la razón por la cual estas adolescentes deciden contraer un embarazo, sin haber completado el nivel de estudio secundario y en algunos casos ni siquiera el primario, comprometiendo así la mejora en su calidad de vida.

Analizadas todas las variables, se puede apreciar, en fin, que las mujeres entre 13 y 24 años encuestadas que asisten a la consulta en el Hospital de Concepción de la Sierra presentan las siguientes características:
- Edad promedio de 18, 4 años.

- En gran porcentaje, tienen una unión de pareja estable.

- Poseen un bajo nivel educacional; la mayoría solamente con primaria completa.

- Un pequeño grupo posee alguna cobertura social.

- La mayoría habita una residencia propia, con los servicios básicos satisfechos (luz eléctrica y agua potable).

- Conocen los métodos anticonceptivos y la mayoría los usa o los usó alguna vez.

- Con respecto a los métodos anticonceptivos, el más utilizado es la píldora anticonceptiva: el $78 \%$ lo retira del hospital.

- De las pacientes, 80\% recibió información acerca de los anticonceptivos por parte del médico que se lo prescribió.

- Al momento de la encuesta, más del 50\% no estaba embarazada, aunque todas presentaban por lo menos una gesta previa, de las cuales la gran mayoría buscó el embarazo.

- De las pacientes que dijeron no haber buscado el embarazo, casi el $80 \%$ mencionó no haber usado método anticonceptivo alguno. Caso similar ocurrió con las pacientes embarazadas al momento de la encuesta.

- La mayoría de las pacientes cuenta con el salario universal por hijo como principal ingreso económico.

\section{Agradecimientos}

Al Director del Hospital Nivel 1 de Concepción de la Sierra, Dr. César Báez, por permitirme realizar el estudio en mi etapa de residencia. Al personal del servicio de Farmacia donde realicé mi rotación de baja complejidad y llevé a cabo el trabajo. A las promotoras de salud del hospital, que me ayudaron con el llenado de las encuestas. 


\section{Referencias}

1. Díaz A, Sugg C, Valenzuela M. Embarazo en la adolescencia. Educación sexual y anticoncepción previa. Rev. SOGIA. 2004; 11(3): 79-83.

2. Carreón VJ, Mendoza SH, Pérez HC, Gil AI, Soler HE, González SR. Factores económicos asociados al embarazo en adolescentes. Archivos en Medicina Familiar. 2004; 6 (3): 70-73.

3. Soto MO, Franco BA, Franco BA, Silva VJ, Velázquez ZG. Embarazo en la adolescencia y conocimientos sobre sexualidad. Rev. Cubana Médica Gen Integr. 2003; 19(6): 0-0.

4. Della MM, Landoni A. Uso de métodos anticonceptivos e información sexual en relación con los antecedentes de aborto en un muestra de adolescentes embarazadas de 13 a 18 años, escolarizadas, de la ciudad de Buenos Aires. Rev. Hosp. Mat. Inf. Ramón Sardá. 2003; 22 (1): 3-10.

5. De Dios MA, Medina MR. Qué saben las adolescentes acerca de los métodos anticonceptivos y cómo los usan. Estudio de una población adolescentes de Piedras Blancas. Rev. Med. Urug. 2006; 22 (3): 185-190.

6. Pomata J, García H, Otheguy L, Aspres N, Fernández T. Adolescencia y embarazo. Revista Hospital Materno Infantil Ramón Sarda. 1997. 16 (2): 52-56.

7. Molina M, Ferrada C, Pérez R, Cid L, Casanueva V, García A. Embarazo en la adolescencia y su relación con la deserción escolar. Rev. Med. Chile. 2004; 132 (1): 65-70.

8. Rivero MI, Schinini J, Feu MC, Gonzalez E, Villalba MT. Adolescencia y embarazo: ¿es un factor de riesgo? Revista Médica del Nordeste. 2002; 3: 39-42.

9. González GC, Rojas MR, Hernández SM, Olaiz FG. Perfil del comportamiento sexual en adolescentes mexicanos de 12 a 19 años de edad: resultados de la ENSA 2000. Salud pública Méx. 2005; 47 (3): 209-218.

10. Cruz HJ, Yanes QM, Isla VA, Hernández GP, Velasco BA. Anticoncepción en la adolescencia. Rev Cubana Endocrinol. 2007; 18(1):0-0.

11. Pérez PZ, Casas RL, Peña GL, Miranda RO, Zaldívar GM. Información sexual en un grupo de adolescentes. Rev. Cubana Med Milit. 2002; 31(4):0-0.

12. Núñez UR, Hernández PB, García BC, González D, Walker D. Embarazo no deseado en adolescentes, y utilización de métodos anticonceptivos posparto. Salud pública Méx. 2003; 45 (supl 1): s92s-102.

13. Santín VC, Torrico LE, López LJ, Revilla DC. Conocimiento y utilización de los métodos anticonceptivos y su relación con la prevención de enfermedades de transmisión sexual en jóvenes. Anales de Psicología. 2003; 19 (1): 8190 .

Cienc. innov. salud. Diciembre 2013; 1 (2):114 - 119. Universidad Simón Bolívar (Col). ISSN: 2344-8636 http://portal.unisimonbolivar.edu.co:82/rdigital/innovacionsalud 